Rev. Elev. Méd. vét. Pays trop., 1978, 31 (4) : 423-426.

\title{
La sarcosporidiose chez le buffle africain (Syncerus caffer)
}

\author{
par Ch. PERROTIN (*), M. GRABER (*), J. THAL (**), J. P. PETIT (***)
}

RESUMÉ

\begin{abstract}
Après quelques généralités sur la sarcosporidiose, une description des kystes sarcosporidiens trouvés chez le buffle africain ( $S$. caffer) et quelques hypothèses sur le cycle évolutif sont données.
\end{abstract}

\section{CONSIDÉRATIONS GÉNÉRALES}

La sarcosporidiose est une protozoose qui affecte un nombre important d'espèces parmi toutes les classes de vertébrés.

Cette affection, qui atteint surtout les herbivores, est due à un protozoaire de nature coccidienne, appartenant au genre Sarcocystis (Apicomplexa). Elle se traduit par la formation et la localisation aux muscles striés et lisses de kystes macro ou microscopiques.

Ces kystes, de structure variable selon l'espèce de sarcosporidie, renferment les corpuscules de Rainey ("germes infectieux d'origine interne ») en forme de banane caractéristique (mérozoïtes $=$ bradyzoïtes).

Le cycle évolutif de ce parasite nécessite (en l'état actuel des connaissances) l'intervention de 2 hôtes:

Un herbivore qui héberge les kystes et joue le rôle d'hôte intermédiaire (proie), un carnivore le plus souvent qui ingère les mérozoïtes contenus dans les kystes, et émet dans le milieu extérieur

(*) Service de Parasitologie. Ecole Nationale Vétérinaire de Lyon. Route de St-Bel, 69260 Marcy L'EtoileCharbonnières Les Bains.

(**) Institut d'Elevage et de Médecine Vétérinaire des Pays Tropicaux, 10, rue Pierre-Curie, 94700 MaisonsAlfort.

(***) Directeur de Recherche à l'Institut d'Elevage et de Médecine Vétérinaire des Pays Tropicaux, 10, rue Pierre-Curie, 94700 Maisons-Alfort. des sporocystes infectants. Ce carnivore dans l'intestin duquel le cycle évolutif coccidien se déroule, joue le rôle d'hôte définitif (prédateur).

Ces kystes sarcosporidiens sont décrits chez de nombreux herbivores sauvages de la faune africaine, et notamment chez le buffle (Syncerus caffer) en Afrique Orientale à plusieurs reprises, en Tanzanie (5), en Ouganda (5), en Afrique du Sud (9).

L'infestation semble fréquente (47 p. 100 selon BINDERNAGEL), bien que la statistique soit faite sur un nombre réduit d'animaux.

Des autopsies réalisées sur des buffles abattus en Afrique centrale (Empire Centrafricain) ont révélé à plusieurs reprises la présence de kystes sarcosporidiens que nous nous proposons d'étudier ici.

\section{MATÉRIEL ET MÉTHODE}

\section{A) MATÉRIEL}

Les prélèvements proviennent de 42 buffles dont l'âge varie entre 4 et 12 ans. Ils ont été abattus au nord de la localité de Bangassou, dans un périmètre limité par les $6^{\mathrm{e}}$ et $8^{\mathrm{e}}$ parallèles, et les $23^{\circ}$ et $24^{\circ}$ degrés de longitude. Ces échantillons sont fixés au formol depuis 19701971. Les prélèvements correspondant à 5 animaux sont inutilisables. Les prélèvements effec- 
tués sur les 37 buffles restants sont au nombre de 41 et se répartissent de la façon suivante :

Nombre de prélèvements selon l'origine

$\begin{array}{ccc}\text { Langue } & \text { Esophage } & \text { Cour } \\ 17 & 14 & 1 \\ \text { Diaphragme } & \text { Muscles } & \text { Total } \\ 6 & 3 & 41\end{array}$

Au total, 55 animaux ont été abattus sur cette aire, 42 se révèlent porteurs de kystes sarcosporidiens, ce qui donne un pourcentage d'animaux infestés de 76,3 p. 100 (les animaux abattus étant considérés comme répartis au hasard).

\section{B) MÉTHODE}

- Dans un premier temps, un kyste de chaque prélèvement est ouvert, un étalement coloré au May Grunwald Giemsa est réalisé.

Pour chaque lame, 50 corpuscules de Rainey sont mesurés (longueur et largeur) pour déterminer leurs dimensions. Pour cela nous utilisons un objectif à immersion $(\times 100)$ et une lame micrométrique (une graduation $=1,7 \mu$ ). La mesure peut être effectuée à une demi-graduation près.

\section{RẼSULTATS}

A) Les kystes macroscopiques, peu enchâssés dans le tissu musculaire sont volumineux et de taille variable, allant pour la majorité, de la taille d'un grain de riz à celle d'un haricot. Le nombre des kystes est important dans les prélèvements qui sont à notre disposition (plusieurs dizaines). Ils sont le plus souvent de forme allongée, quelques-uns sont globuleux, ressemblant à un grain de maïs.

Macroscopiquement, les kystes que nous avons observés correspondent à la description de KALINER (5).

$B$ ) A l'ouverture, ces kystes renferment une substance blanc nacré granuleuse qui s'étale difficilement.

C) Les étalements colorés au MGG révèlent la présence de corps en forme de banane, colorés en mauve. Le noyau se localise au pôle le plus large. A l'autre pôle on note des granulations plus ou moins volumineuses. Des mesures de ces éléments sont effectuées, et après analyse statistique, il s'avère que :
- Pour un individu; les parasites recueillis au niveau de la langue, sont légèrement plus petits $(11,28 \mu$ sur $3,00 \mu)$ que ceux recueillis au niveau de l'œsophage $(11,67 \mu$ sur $3,12 \mu)$. La variation est de $+3,5$ p. 100 en longueur et de +4 p. 100 en largeur.

Ces variations se retrouvent pour chaque individu d'un ensemble.

- Des variations significatives de taille existent également entre différents individus, et pour un même organe parasité (langue).

Les mesures moyennes extrêmes rencontrées sont :

$$
\begin{aligned}
& \text { Longueur : } 13,43 \mu \text { et } 8,5 \mu . \\
& \text { Largeur : } 2,85 \mu \text { et } 3,4 \mu .
\end{aligned}
$$

La précision statistique obtenue au seuil de 95 p. 100 tient compte, dans l'évaluation, de la taille des parasites (voir annexe) :

- de la précision permise par l'appareillage utilisé ;

- de l'hétérogénéité des parasites prélevés sur un seul organe d'un même individu.

D) Structure des kystes :

L'examen des coupes histologiques réalisées sur notre matériel ne révèle pas de microkystes tels que KALINER les décrit.

Les coupes transversales, ovales ou rondes, sont de $3,2 \mathrm{~mm}$ de diamètre en moyenne pour les kystes de la langue, et de $2,6 \mathrm{~mm}$ pour ceux de l'œsophage. Ils sont limités par une membrane externe d'épaisseur moyenne 15-20 $\mu$. Cette membrane émet vers l'intérieur du kyste des cloisons qui délimitent des logettes (Photos $\mathrm{n}^{\text {os }} 1$ et 2 ). Au voisinage immédiat de la membrane limitante, les logettes sont petites et renferment quelques cellules arrondies, volumineuses à noyau excentré. Ces cellules correspondant à des formes immatures de sarcosporidies sont les métrocytes.

Les logettes augmentent de volume et sont bourrées de formes en banane ou bradyzoïtes. Vers le centre du kyste, la paroi des logettes s'amincit, et les germes infectieux sont très peu nombreux (Photo $n^{\circ}$ 2) voire absents la plupart du temps. KALINER (4) fait la même observation $« \ldots$ the center of larger cysts were free from parasites $»$.

Nous n'avons parmi les préparations à notre disposition jamais noté de réaction inflammatoire au voisinage immédiat des kystes. 
Photo $\mathrm{n}^{\circ}$ 1. - Détail de la paroi et ogette.
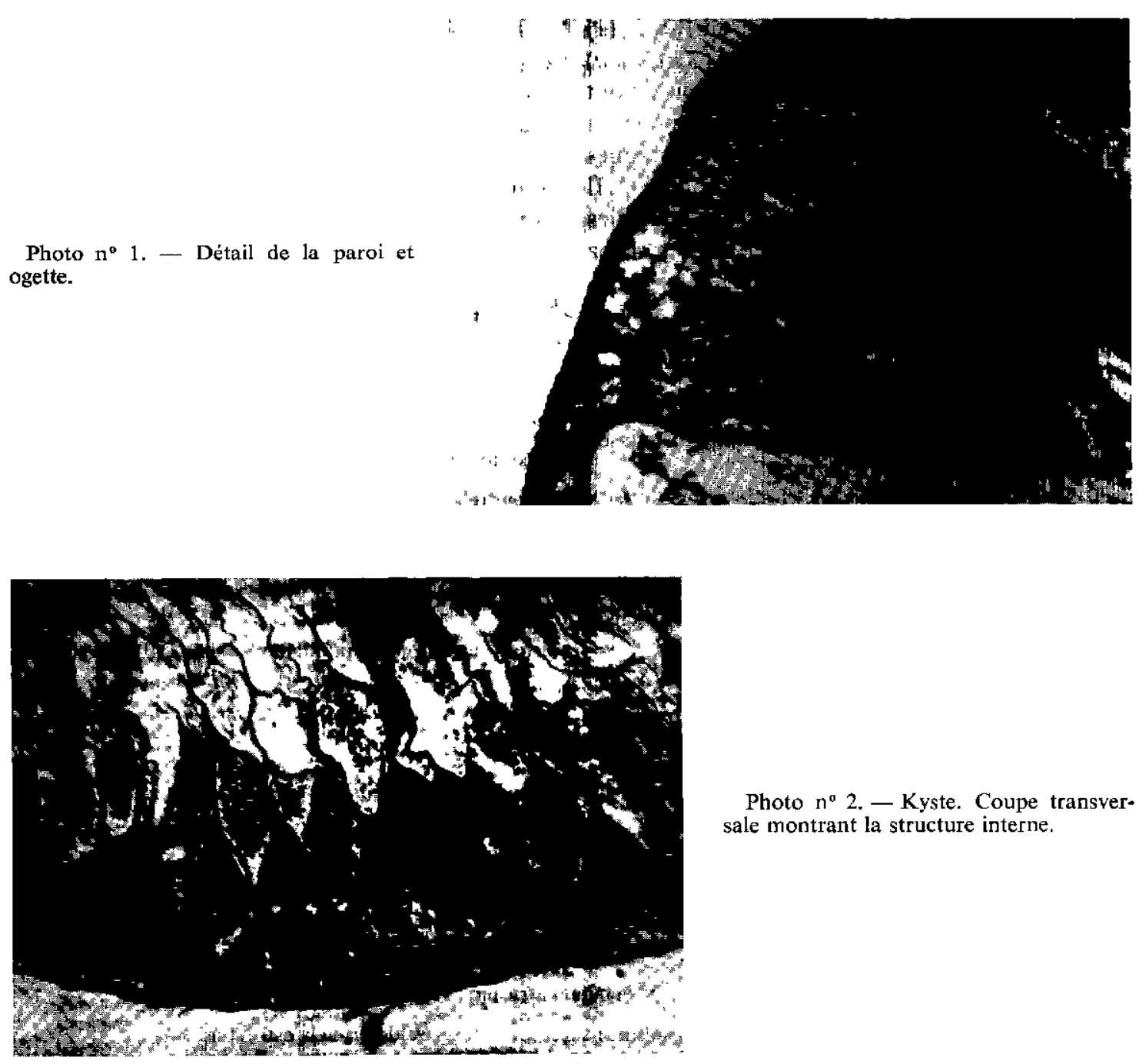

Photo $\mathrm{n}^{\circ}$ 2. - Kyste. Coupe transversale montrant la structure interne.

\section{DISCUSSION}

A) Actuellement, aucune description de la structure des kystes sarcosporidiens rencontrés chez le buffle n'est donnée. Celle que nous donnons ici se rapproche de certains kystes trouvés chez diverses espèces d'antilopes, ou d'animaux domestiques. Un point commun est l'absence ou le nombre réduit de germes infectieux dans la partie centrale des kystes les plus âgés.

$B)$ Les variations de taille notées sur les corpuscules de RAINEY peuvent indiquer :

- L'existence d'espèces différentes de sarcosporidies chez un même animal avec des localisations préférentielles pour tel ou tel organe ;

- Des modifications de taille liées au tissu parasité (fibres striées pour la langue et fibres lisses pour l'œsophage).

Cet aspect morphologique méritait d'être signalé car il est probable que les animaux sauvages (comme les animaux domestiques) sont capables d'héberger plusieurs espèces de sarcosporidies.

C) Dans l'introduction de ce texte, il est signalé que le cycle évolutif des sarcosporidies nécessite l'intervention de deux hôtes.

Il serait donc intéressant de rechercher les hôtes définitifs parmi les prédateurs du buffle qui, dans la région intéressée, sont principalement : le lion, la hyène, le chacal, ces deux dernières espèces vivent dans des biotopes voisins. Notons ici que des coccidies ont été décrites chez des carnivores sauvages mais en captivité.

Il est possible qu'un oiseau soit hôte définitif. 
Ce type de cycle est décrit par CERNA (1) entre la souris et la chouette effraie (Tyto alba) en Europe. Les vautours en milieu tropical sont des prédateurs importants des animaux morts, et ils consomment préférentiellement les organes les plus parasités (langue et cesophage). Il serait donc intéressant d'étudier les coccidies chez ces espèces, et leur éventuel passage chez les grands ruminants. Le pourcentage d'animaux atteints de sarcosporidiose est élevé, ceci suppose donc une grande dissémination des formes infestantes. Si les oiseaux n'interviennent pas comme hôtes définitifs, il n'est pas interdit de penser qu'ils jouent un rôle dans la dissémination des formes infestantes.

En l'état actuel des connaissances, il est impossible de connaître le cycle évolutif. Il faudrait pour cela infester expérimentalement les hôtes définitifs les plus probables avec des kystes frais prélevés sur des buffles.

Les espèces de sarcosporidies se définissent essentiellement par les deux hôtes intervenant dans leur cycle, ce qui permet leur classification. Aussi éviterons-nous de donner une dénomination aux sarcosporidies décrites chez le buffle, afin de ne pas obscurcir une classification déjà complexe.

\section{ANNEXE}

\section{Nature de la précision}

Ecart-type pour l'évaluation des dimensions d'un parasite ....

Précision correspondante .....

Erreur relative correspondante.

Précision de la moyenne des dimensions caractéristiques d'un individu (sur 50 évaluations) $\ldots \ldots \ldots \ldots \ldots \ldots \ldots$

Erreur relative correspondante.

$\begin{array}{cc}\text { Longueur } & \text { Largeur } \\ - & - \\ 1,00 & 0,42 \\ +2 & +0,8 \\ 30 \text { p. } 100 & 45 \text { p. } 100 \\ & \\ +0,3 & +0,12 \\ 4,2 \text { p. } 100 & 6,6 \text { p. } 100\end{array}$

\section{SUMMARY}

\section{Sarcosporidiosis in african buffaloe (Syncerus caffer)}

After a review of sarcosporidiosis, a description of sarcosporidian cysts found in african buffaloe ( $S$. caffer) is given. Some hypothesis about life cycle are proposed.

\section{RESUMEN}

La sarcosporidiosis en el búfalo africano (Syncerus caffer)

Después de una revisión sobre la sarcosporidiosis, se descrıbe la estrúctura del quiste sarcosporidico encontrado en el búfalo africano (S. caffer). Algunas hypothesis son emitidas à propósito del ciclo evolutivo.

\section{BIBLIOGRAPHIE SOMMAIRE}

1. CERNA (Z.). Cycle de développement sarcosporidien d'une coccidie chez la souris après infestation des animaux par des ookystes. Sporocystes isolés de l'intestin de la chouette effraje. Protistologica, 1977, 13 (3): 401-405.

2. HEYDORN (A. O.), GESTRICH (R.), MEHLHORN (H.), ROMMEL (M.). Proposal for a new nomenclature of the Sarcosporidia. Z. Parasitkde, 1975, 48 (2): 426.

3. HSI WANG. Note on bovine sarcosporidiosis. J. $\mathrm{Pa}$ rasit., 1950, $36: 416-422$.

4. KALINER (G.). Observations on the morphology of Sarcosporidian cysts of some East African game animals (Artiodactylae). Z. Parasitkde, 1975, 46:1323.
5. KALINER (G.), SACHS (R.), FAY (L. D.), SCHIEMANN (B.). Untersuchungen über das Vorkommen von Sarcosporidian beiostafrikanischen Wildtieren. Z. Tropenmed. Parasit. 1971, 2 : 156-164.

6. LEVINE (N. D.). Nomenclature of sarcocystis in the ox and sheep and of fecal coccidia of the dog and cat, $J$, Parasit., 1977, 63 (1) : 36-51.

7. MUNDAY (B. L.), RICKARD (M. D.). Is Sarcocystis tenella two species? Aust. Vet. J, 1974, 50 (12) : 558-559.

8. THILLS (E.), DEOM (J.), FAGARD (P.). Considérations sur la sarcosporidiose au Katanga (Congo Belge). Bull. Soc, Path. exot., 1960, 53 : 106-110.

9. THORNTON (H.). Sarcosporidiosis : a review. Trop. anim. Hlth. Prod., 1972, 4 : 54-57. 